Sylvain DAVID, «"Héros négatifs" et communauté(s) du refus dans l'œuvre de Cioran », dans Y. HAMEl et M. Bouchard (dir.), Portrait de l'homme de lettres en héros, @nalyses, hiver 2006

\title{
Sylvain DAVID
}

\section{« Héros négatifs» et communauté(s) du refus dans l'œuvre de Cioran}

L'essayiste français d'origine roumaine Emil Cioran (1911-1995) a souvent apparenté sa démarche à une forme d'héroïsme. Dès son premier livre, Sur les cimes du désespoir (publié en roumain en 1933), il évoque un "hérö̈sme de la résistance et non de la conquête, qui se manifeste par la volonté de se maintenir sur les positions perdues de la vie» (1995, p. 34) De même, dans le fragment «Penseurs crépusculaires » du recueil Précis de décomposition (ouvrage avec lequel il entame sa carrière française, en 1949), l'essayiste remarque : «Et si nous sommes les héros négatifs d'un Âge trop mûr, par ce fait même nous en sommes les contemporains: trahir son temps ou en être le fervent, exprime - sous une contradiction apparente - un même acte de participation. » (1995, p. 612) Cette dernière citation est particulièrement intéressante, car elle situe la thématique du héros dans une perspective sociale, elle enrichit la vision romantique propre aux textes de jeunesse de l'écrivain de considérations sur une philosophie pessimiste de l'histoire.

Deux détails posent cependant problème dans cet extrait. Tout d'abord, pourquoi un essayiste qui dénonce inlassablement les fauxsemblants et les illusions ressent-il le besoin de se définir au travers d'une figure métaphorique — d'un personnage de fiction - comme le «héros négatif»? Quelle résonance son œuvre retire-t-elle d'une pareille médiation ou mise en scène? Ensuite - et surtout pourquoi cette revendication d'une attitude d'opposition et de refus s'énonce-t-elle au "nous », c'est-à-dire au nom d'un groupe, d'une collectivité? Si le héros est généralement garant de la communauté ou de lui-même s'il est "problématique» (Lukács) — que penser d'une communauté de héros, "négatifs » de surcroît? S'agit-il d'une contestation collective, d'une négation commune, ou, à l'inverse, d'un simple agglomérat de marginaux, individuellement désenchantés, 
Sylvain DAVID, «"Héros négatifs" et communauté(s) du refus dans l'œuvre de Cioran », dans Y. HAMEl et M. Bouchard (dir.), Portrait de l'homme de lettres en héros, @nalyses, hiver 2006

n'ayant en commun que l'exercice de la négativité? C'est à ces questions que tentera de répondre cette étude, avant tout pour tenter de mieux comprendre la posture énonciative particulière de Cioran, mais également, dans une perspective plus large, pour réfléchir à ce que pourrait être le rôle ou la fonction du penseur, au sein de la collectivité, en une époque - cet «Âge trop mûr » que représente la modernité tardive - où des valeurs singulières, individuelles, comme la négativité, l'abstention et le désistement font (la plupart du temps) figure d'ultime forme d'authenticité.

\section{Un héroïsme à rebours}

Tout d'abord, force est de reconnaître que l'expression «héros négatif » n'apparaît qu'une seule fois dans l'œuvre de Cioran, ce qui met les choses en perspective en rappelant qu'il s'agit là non pas de la revendication d'une identité absolue, mais bien de l'une des multiples définitions de soi offertes par l'écrivain. Passé ce premier obstacle, reste la difficulté que l'essayiste (qui ne se soucie pour ainsi dire jamais de définir son lexique) ne précise jamais en quoi pourrait consister un tel hérö̈sme à rebours. Par contre, comme on l'a souligné dans l'introduction, le terme « héros » apparaît fréquemment sous la plume de Cioran (même si, une fois de plus, la signification précise de ce vocable est laissée à l'imagination du lecteur). Plutôt que de tenter d'extrapoler un sens à partir d'un examen systématique de chaque occurrence de ce mot, on partira de la définition offerte par le dictionnaire - définition qu'il sera ensuite possible de renverser, pour en donner à voir le versant négatif — dans la visée de déterminer en quoi ces catégories basiques de compréhension permettent de mieux pénétrer la prose et la pensée de l'écrivain.

Si on se fie au Dictionnaire alphabétique et analogique de la langue française de Paul Robert, le terme "héros » renvoie avant tout à un "personnage légendaire auquel on prête un courage et des exploits remarquables » (1957, p. 494). Pareille définition peut paraittre fort éloignée des écrits de Cioran qui, malgré leur tendance à englober une multitude de thématiques, n'ont que peu à voir avec la mythologie ou l'épopée. Mais c'est justement cette distance qui s'avère significative. En effet, 
Sylvain DAVID, «"Héros négatifs" et communauté(s) du refus dans l'œuvre de Cioran », dans Y. HAMEl et M. Bouchard (dir.), Portrait de l'homme de lettres en héros, @nalyses, hiver 2006

cet «Âge trop mûr» que l'essayiste dresse en toile de fond de son œuvre est justement celui où l'individu, renvoyé à lui-même, ne croit plus en aucune forme d'élévation spirituelle ou de transcendance: " Les mythes redeviennent concepts : c'est la décadence. » (1995, p. 679) Dans un tel contexte, le héros ne peut être que "négatif ", c'est-à-dire amoindri, amenuisé, incapable de se forger une destinée: "Si l'humanité ne se fût amusée à discuter ses propres forces, elle n'eût point dépassé la vision et les modèles d'Homère. Mais la dialectique, en ravageant la spontanéité des réflexes et la fraîcheur des mythes, a réduit le héros à un exemplaire branlant. » $(1995$, p. 682) Vu de cette perspective, le "héros négatif», déchu de sa dimension légendaire, n'est pas loin du citoyen lambda, de l'individu moyen contemporain.

Une figure héroïque, fût-elle passive et diminuée, ne saurait toutefois se confondre entièrement avec le commun. De fait, une seconde définition du mot "héros » évoque "celui qui se distingue par ses exploits ou un courage extraordinaire » (1957, p. 494). Là encore, une telle compréhension du concept parait fort éloignée des préoccupations de Cioran, du moins jusqu'à ce qu'on la renverse, qu'on en explore le versant négatif. Si l'on part du principe, posé précédemment, que le savoir critique a miné la grandeur de l'humanité, que « [] a psychologie est le tombeau du héros» (1995, p. 682), on ne peut faire autrement que d'aboutir à la conclusion que la seule épreuve qui s'offre encore à l'individu moderne est celle d'entrer encore davantage en lui-même, de pousser le plus loin possible dans la connaissance de soi. Ainsi, l'« héroïsme négatif » pratiqué par Cioran serait celui du renoncement, et consisterait, par l'exercice de la pensée critique, à sciemment se détourner de toute forme de grandeur.

La véritable existence tragique ne se rencontre presque jamais parmi ceux qui savent manier les puissances secrètes qui les harassent; à force d'amoindrir leur âme par leur œuvre, où puiseraient-ils l'énergie d'atteindre l'extrémité des actes? Tel héros s'est accompli dans une modalité superbe du mourir parce qu'il lui manquait la faculté de s'éteindre progressivement dans des vers. Tout héroïsme expie - par le génie du cœur - un talent en défaut, tout héros est un être sans talent. (voir 1995, p. 626) 
Sylvain DAVID, «"Héros négatifs" et communauté(s) du refus dans l'œuvre de Cioran », dans Y. HAMEl et M. Bouchard (dir.), Portrait de l'homme de lettres en héros, @nalyses, hiver 2006

L'intelligence, la sensibilité et la délicatesse se voient ici - thème cioranien récurrent - posés comme gages de faiblesse. Mais aussi, et surtout, le véritable héroïsme de l'homme de lettres est présenté comme étant la volonté de persister à écrire, à s'exprimer, au détriment de sa personne, à l'encontre de sa propre substance vitale.

Un tel épuisement de soi par la voie de l'écriture est-il admirable? La question peut paraître étrange, mais n'en renvoie pas moins à la troisième définition du héros: «tout homme digne de l'estime publique, de la gloire par sa force de caractère, son génie, son dévouement total à une cause, une œuvre... » (1957, p. 495) Si l'idée d'une certaine abnégation face à la pensée et la littérature demeure tout à fait en accord avec la tonalité générale des écrits de Cioran, celle de se poser en modèle pour ses concitoyens l'est beaucoup moins. De fait, celui qui a pu déclarer "Plutôt dans un égout que sur un piédestal» (1995, p. 1342) tend à évoquer, une fois de plus, une acception négative du terme, sa compréhension à rebours. Cela dit, il est frappant de constater que l'essayiste lui-même consent à établir une gradation des épreuves, à dresser un palmarès de l'exploit au sein de cet univers particulier qui est le sien. D'où un paradoxal éloge du « héros de la paresse totale » (1995, p. 619):

Car le Sage - théoricien de la mort limpide, héros de l'indifférence et symbole de la dernière étape de la philosophie, de sa dégénérescence et de sa vacuité - a résolu le problème de sa propre mort... et a supprimé dès lors tous les problèmes. Pourvu de ridicules plus rares, il est un cas limite, que l'on rencontre à des périodes extrêmes comme une confirmation exceptionnelle de la pathologie générale. (1995, p. 611)

Suivant cette idée, l'apposition du qualificatif "négatif» au vocable généralement noble d'« hérö̈sme » ne renverrait pas seulement à une défaillance généralisée, sociale (comme cela a pu être le cas jusqu’à présent), mais également à une impuissance personnelle de la part de Cioran, qui avoue viser sans cesse à ce type de «sagesse » sans jamais pour autant y parvenir. 
Sylvain DAVID, «"Héros négatifs" et communauté(s) du refus dans l'œuvre de Cioran », dans Y. HAMEl et M. Bouchard (dir.), Portrait de l'homme de lettres en héros, @nalyses, hiver 2006

Cette dernière remarque permet d'introduire une ultime manière de comprendre l'idée du «héros» en tant que "personnage principal d'une œuvre littéraire, dramatique, cinématographique...» (1957, p. 495) Car il est évident que, sur ce point, l'œuvre cioranienne pose problème: alors qu'à la lecture d'un roman, il est désormais automatique de distinguer entre auteur et narrateur, créateur et personnage, que penser d'une œuvre de réflexion (donc de prime abord non fictive) où l'écrivain parle en son propre nom tout en s'affublant de surnoms imagés tels «un douteur en transe, un fanatique sans credo, un héros de la fluctuation » (1995, p. 1450) ou, bien sûr, un " héros négatif »? Une première piste de réponse à ce sujet serait d'avancer que, comme l'écrivain retient de l'univers une vision essentiellement métaphorique - l'expression « un Âge trop mûr » en est le meilleur exemple -, il ne peut faire autrement que de se présenter de la même manière (ton et registre similaires), afin de convenablement s'y situer: on remarquera d'ailleurs que l'apposition d'un nom positif et d'un adjectif négatif (" héros négatif ») correspond au procédé général de composition de l'œuvre ("syllogismes de l'amertume », "mauvais démiurge », etc.). Une autre manière de comprendre cette définition particulière qu'offre Cioran de sa propre personne serait d'y voir la mise en place d'un ethos, la revendication d'une capacité de locuteur à l'image de la vision pessimiste transmise par ses écrits. Mais ce dernier point implique l'idée d'une relation ou d'un contact avec le lecteur, ce à quoi l'on reviendra dans la section suivante.

Bref, le concept de «héros négatif», chez Cioran, renvoie au dilemme de celui qui, victime de la rationalité desséchante de l'ère de la connaissance de soi, en vient à lutter contre l'ultime ennemi à abattre : lui-même. Le panthéon personnel de l'essayiste — composé entièrement de suicidés de la littérature - est, à ce sujet, particulièrement révélateur : "Lorsqu'on est jeune on se cherche des héros : j'ai eu les miens : Henri de Kleist, Caroline de Guenderode, Gérard de Nerval, Otto Weininger...» (1995, p. 732) De prime abord, cette aspiration à "trahir son temps » alors même qu'il sait en être le «contemporain » range Cioran parmi ceux qu'Antoine Compagnon nomme les antimodernes, ou «modernes malgré eux » (2005, p. 7). Il est 
Sylvain DAVID, «"Héros négatifs" et communauté(s) du refus dans l'œuvre de Cioran », dans Y. HAMEl et M. Bouchard (dir.), Portrait de l'homme de lettres en héros, @nalyses, hiver 2006

dès lors facile de comprendre en quoi l'épreuve de fuir ce que l'on est - ou, pour reprendre les mots de l'auteur, de «penser contre soi» (1995, p. 821) - constitue, de par son haut niveau de difficulté, une forme d’héroïsme, fût-il négatif. L'essayiste se distingue cependant en énonçant une telle revendication d'individualité au "nous ", c'est-àdire au nom d'un ensemble plus vaste. La question se pose donc : quel type de communauté — héroïque? négative? — le penseur tente-t-il de constituer, et avec qui?

\section{Héros ou prophète?}

Si les remarques qui précèdent permettent de mieux saisir comment se constitue la figure "héroïque » de Cioran au sein de ses écrits, elles n'expliquent toutefois en rien comment celle-ci en vient à acquérir une dimension interpersonnelle, collective. Ce curieux phénomène tend à s'expliquer par le fait que le penseur manifeste une prégnance particulière dans son œuvre : lors même que le texte traite d'un thème en apparence abstrait, la personne de l'essayiste — ou, à tout le moins, son incarnation « littéraire»- demeure constamment en filigrane. De cette manière, plus encore qu'une présence, l'essayiste arrive à développer un effet de proximité, si ce n'est de complicité avec son lecteur. À ce sujet, il importe, afin de bien respecter la dynamique de l'œuvre, de commencer par établir un constat d'ordre général — c'està-dire concernant l'ensemble des écrits — pour seulement ensuite revenir à la question de l'« hérö̈sme négatif » de l'auteur.

Le pacte particulier que noue l'essayiste avec qui se plonge dans son œuvre repose, entre autres, sur un usage virtuose des pronoms personnels : l'expression passe ainsi — souvent dans l'espace d'un même fragment — du « je » au «nous » et au « on » (voire au «tu », au «vous» et au «il»). Bien qu'il soit évident que, pour un styliste comme Cioran, un tel procédé permet des effets de variation, représente une façon de se renouveler, il reste le fait que cette oscillation entre diverses perspectives énonciatives entraîne une série d'effets sémantiques équivoques. De fait, l'exemple le plus représentatif de ce type de déplacement réside dans le constant recours au "nous» effectué dans le texte, comme en témoigne une 
Sylvain DAVID, «"Héros négatifs" et communauté(s) du refus dans l'œuvre de Cioran », dans Y. HAMEl et M. Bouchard (dir.), Portrait de l'homme de lettres en héros, @nalyses, hiver 2006

phrase du type «nous sommes les héros négatifs». L'emploi de la première personne du pluriel, si l'on s'y cantonne, évoque le style universitaire, l'écriture académique, et peut ainsi se faire un gage de la neutralité ou de l'objectivité du locuteur. Or, lorsque — comme le fait Cioran - on saute continuellement d'un pronom à l'autre, l'effet d'alternance a pour conséquence de redonner à chacun de ceux-ci sa fonction grammaticale première. Dès lors, le «nous» reprend sa propriété d'assimilation: on ne parle plus en son nom propre mais par rapport à une communauté.

Dans le contexte global de l'œuvre de l'essayiste, toutefois, le phénomène conserve une part d'ambiguïté dans la mesure où le Précis de décomposition dénonce vertement toute aspiration à la parole collective :

Le pluriel implicite du «on» et le pluriel avoué du «nous» constituent le refuge confortable de l'existence fausse. [...] Le triomphe de la non-authenticité s'accomplit dans l'activité philosophique, cette complaisance dans le «on», et dans l'activité prophétique (religieuse, morale ou politique), cette apothéose du «nous ». (1995, p. 595)

Suivant cette idée, l'usage fait par le penseur du "nous» contrairement à celui qu'en fait Nietzsche, par exemple, qui s'adresse généralement à un ensemble - relève essentiellement du bilan, à l'instar d'un «nous, les modernes », ou, plus généralement, d'un «nous, les humains ». S'il réfute toute propension au discours public, à la harangue, l'écrivain ne parle pas moins fréquemment en fonction d'une entité plus vaste. Par exemple: «Formés à l'école des velléitaires, idolâtres du fragment et du stigmate, nous appartenons à un temps clinique où comptent seuls les cas. » (1995, p. 745) Or, comme quiconque pénètre dans l'œuvre de Cioran participe, selon toute probabilité, des catégories mentionnées ci-dessus — être "moderne » ou être " homme», au sens philosophique du terme l'emploi massif du «nous» effectué par l'écrivain se traduit par un effet de reconnaissance et de participation, par une impression de communauté ressentie par le lecteur. 
Sylvain DAVID, «"Héros négatifs" et communauté(s) du refus dans l'œuvre de Cioran », dans Y. HAMEl et M. Bouchard (dir.), Portrait de l'homme de lettres en héros, @nalyses, hiver 2006

D'où le pacte particulièrement solide que l'essayiste scelle, sans avoir l'air d'y toucher, avec qui le lit: comme ses écrits se veulent une dénonciation de la misère humaine, une réfutation des fictions ambiantes, le recours à la première personne du pluriel se fait, dans l'ensemble, la marque d'un statut d'initié, l'emblème ténébreux de «nous qui avons compris». Cette connivence des détrompés comporte toutefois ses limites. Après avoir dressé un bilan de l'ordre $\mathrm{du}$ «nous», le penseur tend la plupart du temps à changer de perspective pour rappeler, cette fois au singulier, à quel point son cas particulier diffère de celui du reste de l'humanité, si désenchantée soitelle. En témoigne ce collage de citations extraites de "Généalogie du fanatisme ", incipit du Précis de décomposition:

En elle-même toute idée est neutre, ou devrait l'être; mais l'homme l'anime, y projette ses flammes et ses démences; impure, transformée en croyance, elle s'insère dans le temps, prend figure d'événement : le passage de la logique à l'épilepsie est consommé... [...] Idolâtres par instinct, nous convertissons en inconditionné les objets de nos songes et de nos intérêts. [...] Je me sens plus en sûreté auprès d'un Pyrrhon que d'un saint Paul, pour la raison qu'une sagesse à boutades est plus douce qu'une sainteté déchaînée. [...] Il me suffit d'entendre quelqu'un parler sincèrement d'idéal, d'avenir, de philosophie, de l'entendre dire «nous» avec une inflexion d'assurance, d'invoquer les «autres », et s'en estimer l'interprète, - pour que je le considère mon ennemi. (1995, p. 581-583)

Par le biais de ce jeu entre affirmation personnelle et constat collectif, l'écrivain amorce ce qui demeure, avant tout, un dialogue philosophique avec lui-même. Ce faisant, l'essayiste perpétue la pose du " héros négatif » dans la mesure où il ne peut se définir autrement que par opposition à une forme de communauté : il confirme le paradoxe du penseur enraciné dans son époque alors même qu'il aspire à s'en arracher.

Or, par-delà cet éternel soliloque du marginal, le lecteur se voit malgré tout convié à assister à la discussion dans la mesure où, 
Sylvain DAVID, «"Héros négatifs" et communauté(s) du refus dans l'œuvre de Cioran », dans Y. HAMEl et M. Bouchard (dir.), Portrait de l'homme de lettres en héros, @nalyses, hiver 2006

grammaticalement parlant, il ne peut faire autrement que de se sentir concerné par toute proposition formulée au «nous». Dépassant le simple effet de connivence ou de proximité, l'essayiste arrive ainsi à asseoir pleinement sa supériorité sur son lecteur, à maintenir l'autorité et la fascination qu'il peut exercer sur lui alors même qu'il éveille sa curiosité : en laissant entendre qu'il se dissocie de la (supposée) position intellectuelle de celui à qui il s'adresse - ne serait-ce qu'indirectement - le penseur tend, par le fait même, à inciter ce dernier à le suivre. La communauté engendrée est donc une communauté à la fois de proximité et de distance : l'essayiste se met de plain-pied avec son lecteur dans un refus partagé de la masse, mais s'en distingue néanmoins lorsqu'il revendique - comme l'implique la prétention d'être «l'homme-en-dehors-de-tout» (1995, p. 1217) — le fait d'être allé plus loin que tout le monde. L'écrivain communie ainsi avec son lectorat à travers une étape de son cheminement qu'il estime avoir lui-même dépassée.

Cette paradoxale relation avec le lecteur ne va cependant pas de soi, du moins pas autant qu'il n'y paraît à première vue : quelque chose d'insidieux demeure dans la manière de procéder de Cioran. Si sa propension à formuler des sentences à la première personne du pluriel fait en sorte que l'on retire l'impression qu'il dévoile un non-dit collectif, qu'il énonce tout haut ce que chacun pense tout bas, il n'en reste pas moins que ce type de tour rhétorique a pour effet de « souffler » à autrui certaines de ses pensées propres, à les lui présenter comme si elles étaient siennes. Le grand tour de force de Cioran consiste ainsi à faire passer pour des vérités intemporelles, universelles, des affirmations qui lui sont strictement personnelles. Mais quel lien cet effet de glissement sémantique - où l'on ne sait plus exactement qui parle, ou, plutôt, où l'on n'arrive plus à distinguer au nom de qui parle l'essayiste - entretient-il avec la question de l'héroïsme? Cioran, qui semble valoriser la posture du héros au détriment de celle de "prophète », a-t-il véritablement succombé au désir, néfaste selon lui, de parler au nom des autres? 
Sylvain DAVID, «"Héros négatifs" et communauté(s) du refus dans l'œuvre de Cioran », dans Y. HAMEl et M. Bouchard (dir.), Portrait de l'homme de lettres en héros, @nalyses, hiver 2006

\section{Vers l'idée de « communauté négative "}

À l'issue de cet aperçu de l'œuvre cioranienne, où il a été possible tout d'abord de comprendre ce que l'essayiste entend par l'expression "héros négatif», et ensuite de mettre en lumière l'usage particulier qu'il fait des pronoms personnels, il reste à déterminer en quoi l'idée d'un héroïsme à rebours en vient à se décliner au «nous ». Comme il s'agit d'une étude ponctuelle, ayant pour prétexte une expression que l'écrivain n'explicite jamais véritablement, on se bornera à émettre à cet effet une série de trois hypothèses. La première serait que le sentiment de communauté éveillé chez le lecteur par le recours à la deuxième personne du pluriel ne soit qu'un leurre. En effet, à l'époque du Précis de décomposition, Cioran manifeste à maintes reprises son désir de dissoudre la philosophie, de causer l'ultime ruine de la pensée occidentale: «saper l'architecture des leurres sur laquelle s'appuie l'orgueil du penseur et du citoyen [...] - quelle effervescence délicate et sauvage!»(1995, p. 593) De même, il réitère constamment la volonté de produire une œuvre corrosive, exerçant une influence néfaste sur son public: " $[\mathrm{u}] \mathrm{n}$ livre doit remuer des plaies, en provoquer même. Un livre doit être un danger. » (1995, p. 1444) Il faut, bien sûr, à l'image de la constante ironie cioranienne, éviter de prendre de telles affirmations au pied de la lettre, mais, dans leur logique même, n'est-il pas permis d'y déceler le spectre du véritable antihéros, c'est-à-dire du traitre, qui, suivant la logique du cheval de Troie, crée une fausse connivence avec son lecteur afin de mieux ensuite détruire ses illusions?

Une seconde interprétation de cet «héroïsme négatif» énoncé au pluriel serait qu'il s'agit d'une sorte de mécanisme de défense partagé - entre l'essayiste et son public - contre un monde perçu comme étant par trop hostile. Car Cioran, alors même qu'il récuse à quiconque le droit de s'estimer l'interprète de ses semblables, n'en octroie pas moins une licence absolue au créateur, dans la mesure où le délire de ce dernier demeure individuel : «Et celui qui parle au nom des autres est toujours un imposteur. Politiques, réformateurs et tous ceux qui se réclament d'un prétexte collectif sont des tricheurs. Il n'y a que l'artiste dont le mensonge ne soit pas total, car il n'invente que soi. » (1995, p. 594) Or, si on pousse une telle logique à son apogée, pourquoi ne 
Sylvain DAVID, «"Héros négatifs" et communauté(s) du refus dans l'œuvre de Cioran », dans Y. HAMEl et M. BouCHARD (dir.), Portrait de l'homme de lettres en héros, @nalyses, hiver 2006

pas présumer que l'écrivain, après s'être «inventé » lui-même par le biais d'une multitude de surnoms imagés, ait été ensuite tenté d'étendre cette reconfiguration de sa personne à son public, ait voulu se façonner un lectorat à son image? Selon cette conception du «nous» cioranien, la communauté établie avec le lecteur serait ici autant virtuelle que réelle, car l'essayiste se trouverait à partager la recette de son héroïsme particulier avec quiconque se plonge dans son œuvre, à faire de ses écrits le mode d'emploi d'une résistance passive à une époque décevante. Une telle lecture expliquerait, en outre, la tonicité souvent remarquée de l'œuvre cioranienne, qui tranche pourtant avec le pessimisme de son contenu.

L'ultime hypothèse à ce sujet consisterait à avancer que, par-delà les arguments somme toute « littéraires » avancés jusqu'à maintenant, il se crée une véritable communauté de principe entre Cioran et son public, mais que, compte tenu du fait que tout cela se situe dans cet «Âge trop mûr » que représentent les temps modernes, où utopies et valeurs collectives ont de moins en moins cours, écrivain et lecteur ne se rejoignent plus dans ce qu'ils ont en partage, mais au contraire dans ce qu'ils dénoncent. En effet, le penseur lui-même remarque : «Naguère, on se définissait par les valeurs auxquelles on souscrivait; aujourd'hui, par celles que l'on répudie. » (1995, p. 980) Ne serait-il pas dès lors possible d'étendre cette logique à une vision sociale et d'y voir le fondement de ce qu'on pourrait nommer une communauté du refus? Dans cette perspective, tant l'écrivain que son lecteur pourraient prétendre à une forme d'« hérö̈sme négatif» — un combat contre son époque que chacun assumerait à sa manière — pour se rejoindre ensuite non pas dans une action concertée, une pensée commune, mais dans le simple parallélisme de leur démarche. On serait ainsi tenté de conclure la présente réflexion en avançant que le rôle du «penseur crépusculaire » (puisque c'est le titre du fragment cioranien d'où est tirée l'expression "héros négatif») n'est pas, ou plus, d'exprimer au mieux les valeurs et représentations d'une conscience collective, mais plutôt de meubler une absence de consensus, de se constituer en point de polarisation autour duquel peut se constituer, par le biais de la littérature et de l'esthétique, une telle collectivité du refus, une communauté négative. 
Sylvain DAVID, “"Héros négatifs" et communauté(s) du refus dans l'œuvre de Cioran », dans Y. HAMEl et M. Bouchard (dir.), Portrait de l'homme de lettres en héros, @nalyses, hiver 2006

\section{Bibliographie}

CIORAN, Emil. 1995, CEurres, Paris, Gallimard, coll. « Quarto ».

Compagnon, Antoine. 2005, Les Antimodernes, Paris, Gallimard, coll. «Bibliothèque des idées ».

ROBERT, Paul. 1957, Dictionnaire alphabétique et analogique de la langue française. Tome troisième, Casablanca, Société du nouveau Littré / Paris, Société administrative française des œuvres de Paul Robert (S.A.F.O.R.). 